\title{
An Emergency Decision Making Method Based on Prospect Theory for Different Emergency Situations
}

\author{
Zi-Xin Zhang ${ }^{1,2} \cdot$ Liang Wang $^{1,2} \cdot$ Ying-Ming Wang ${ }^{1}$
}

Published online: 11 June 2018

(C) The Author(s) 2018

\begin{abstract}
Emergency decision making (EDM) is an effective way to deal with emergency situations because of its prominent role in alleviating the losses of properties and lives caused by emergency events. It has drawn increasing attention from both governments and academia, and become an important research topic in recent years. Studies show that decision makers are usually guided by bounded rationality under risk and uncertainty conditions. Their psychological behavior plays an important role in the decision making process, and EDM problems are usually characterized by high risk and uncertainty. Thus, decision makers' psychological behavior has been considered in existing EDM approaches based on prospect theory. An emergency event might evolve into different situations due to its dynamic evolution, which is one of the distinctive features of emergency events. This important issue has been discussed in existing EDM approaches, in which different emergency situations are dealt with by devising different solutions. However, existing EDM approaches do not consider decision makers' psychological behavior together with the different emergency situations and the different solutions. Motivated by such limitation, this study proposed a novel approach based on prospect theory considering emergency situations, which considers not only decision makers' psychological behavior, but also different emergency situations in the EDM process. Two examples
\end{abstract}

Ying-Ming Wang

msymwang@hotmail.com

1 Decision Sciences Institute, Fuzhou University, Fuzhou 350116, China

2 Department of Computer Sciences, University of Jaén, Jaén 23071, Spain and related comparison are provided to illustrate the feasibility and validity of this approach.

Keywords Emergency situations - Emergency decision making · Prospect theory · Psychological behavior

\section{Introduction}

Emergencies are defined as events that take place suddenly_-such as earthquakes, air crash, hurricanes, and terrorist attacks - causing or having the possibility of provoking death and injury, property loss, ecological damage, and social hazards (Liu et al. 2016). When an emergency event occurs, emergency decision making (EDM) is an important process that mitigates the losses of properties and lives caused by the event, which is typically characterized by time pressure and lack of information, resulting in potentially serious consequences (Cosgrave 1996; Levy and Taji 2007). Because of its importance in dealing with emergency events, EDM has become an important research topic in recent years (Fan et al. 2012; Liu et al. 2014; Wang et al. 2015, 2016, 2017; Zhou et al. 2017; Sun et al. 2018).

Emergency events can cause different kinds of losses or damages (property losses, casualties, environmental effects, and so on) due to their high complexity, uncertainty, and dynamic evolution. In order to make the emergency response pertinent and effective, it is necessary for the decision makers, who are in charge of the EDM process, to make reasonable decisions to cope with the emergency event immediately.

Different behaviors play important roles in the decision making process, such as decision makers' psychological behavior (Kahneman and Tversky 1979), strategic manipulation behaviors (Dong et al. 2018), experts' non- 
cooperative behaviors (Dong et al. 2016), and so on. Different behavioral experiments (Kahneman and Tversky 1979; Tversky and Kahneman 1985, 1992; Camerer 1998) have shown that decision makers are usually guided by bounded rationality under risk and uncertainty conditions and their psychological behavior plays an important role in the decision making process. Prospect theory, proposed by Kahneman and Tversky (1979), is regarded as the most influential behavior theory to describe decision makers' psychological behavior under risk and uncertainty. It has been studied (Schmidt and Zank 2008; Bleichrodt et al. 2009) and widely applied to solve various decision making problems when considering decision makers' psychological behavior, such as in multi-attribute decision making (Fan et al. 2013), traffic management (Li 2013; Zhou et al. 2014), emergency decision making (Wang et al. 2015, 2017), and portfolio insurance (Dichtl and Drobetz 2011).

The existing EDM approaches based on prospect theory (Fan et al. 2012; Liu et al. 2014; Wang et al. 2015, 2016) have taken decision makers' psychological behavior into account. Among these approaches, the overall prospect value of alternatives is regarded as the only optimal alternative selection rule-the bigger the overall prospect value, the better the alternative is. When the overall prospect value is greater than zero, according to prospect theory, it means that the decision maker feels gain (positive value: gain; negative value: loss) and the corresponding alternative is considered effective for dealing with the emergency situation. When there is more than one alternatives that the overall prospect values are greater than zero (Fan et al. 2012; Liu et al. 2014; Wang et al. 2015,2016 ), which is usually the case, the alternative with the biggest overall prospect value can deal with the emergency situation more successfully. This alternative is usually also the one with the highest input of human and material resources. The existing EDM approaches based on prospect theory regard the cost of an alternative as a criterion in the decision making process. Such an evolution rule on alternatives may lead to the situation that a decision maker takes the alternative with the highest input of human and material resources to cope with an emergency situation that is not so serious, which will result in wasting resources. This is not reasonable and not close to real world situations because of the limited resources and workforces for specific emergency events. Thus, it is necessary to consider how different solutions can be applied to different emergency situations.

There are different approaches (Shu 2012; Zhang and Liu 2012; Qian et al. 2015; Yu et al. 2015) that have taken this issue into account. But they neglected to include decision makers' psychological behavior in the decision making process. Approaches based on prospect theory do take decision makers' psychological behavior into account but neglect different emergency situations in the decision making process.

This study developed a novel EDM method based on prospect theory aiming to overcome these unsatisfactorily addressed issues. This method not only takes decision makers' psychological behavior into account, but also considers different emergency situations and their different solutions. At the same time, this study proposed a new linear programming selection model to select the optimal alternative, which regards the cost of an alternative as the object and the overall prospect value as the constraints, fully considering the efficiency of each alternative in the selection process.

Section 2 briefly introduces prospect theory and some related studies that show the importance of our proposed approach, and Sect. 3 presents the proposed method dealing with decision makers' psychological behavior and different emergency situations. In Sect. 4, two examples of applying our method are provided and a comparison with other approaches is outlined.

\section{Prospect Theory}

Prospect theory was first proposed by Kahneman and Tversky (1979) and later expanded (Tversky and Kahneman 1992). As the most popular behavior economic theory, it describes the way in which people choose between probabilistic alternatives that involve risk when the probabilities of the outcomes are known. According to the theory, people make decisions based on the potential value of losses and gains rather than the final outcome. Prospect theory has been studied and widely used to solve various decision making problems (Bell 1982, 1985; Tversky and Kahneman 1991, 1992; Abdellaoui et al. 2007; Schmidt et al. 2008; Schmidt and Zank 2008, 2012; Wu and Markle 2008; Bleichrodt et al. 2009; Wakker 2010).

Reference point is one key element in prospect theory, and is defined as a neutral position asset or expectation value of people who want to obtain a certain attribute or not to lose it. The value of the reference point is affected by the expectations of people (Kahneman and Tversky 1979) with respect to the predefined amounts of gains or losses regarding different types of attributes. Comparing with the reference point, for the benefit attributes, the higher the final outcome, the more gains the individual feels, while for cost attributes, the lower the final outcome, the more gains the individual feels. For a better understanding of the reference point concept, see Fig. 1 for the example of a cost attribute.

In the cost attribute example, if there is a possibility to lose some money and predefined amounts are USD 5, 10, 


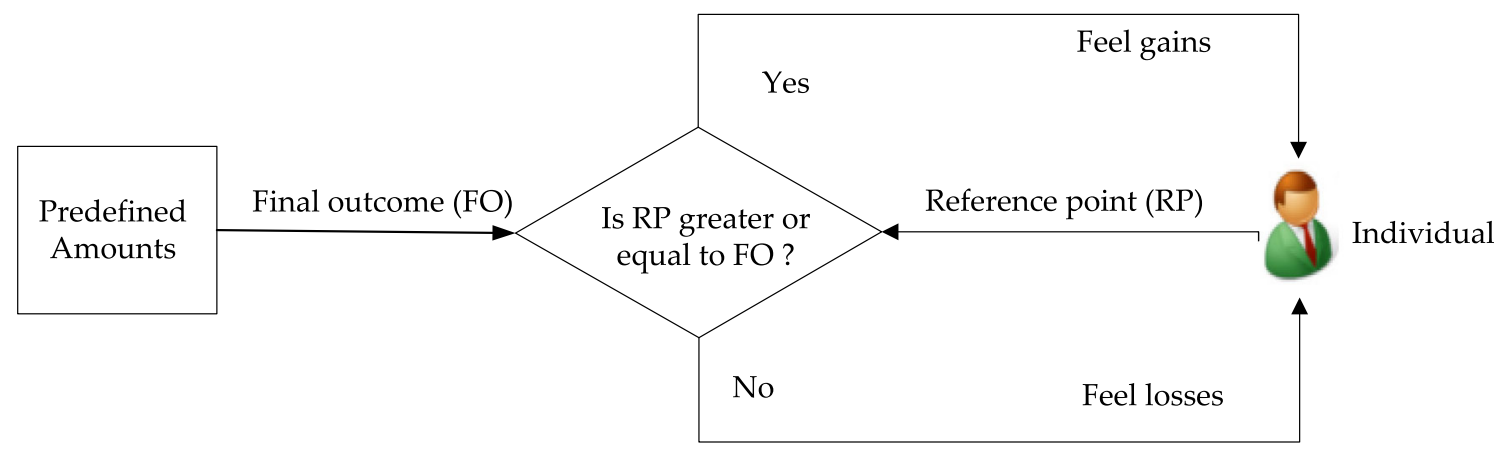

Fig. 1 Gains and losses based on reference point and predefined amounts for a cost attribute

and 20, then assuming that 10 is an acceptable loss amount to an individual (reference point of possible losses), if the final outcome is 5 , he/she feels gains because the final losses are lower than his/her expectation. Benefit attributes can be assessed in a similar way.

Gains and losses are determined by the reference point and the final outcome with respect to different types of attributes. According to Kahneman and Tversky (1979, 1992), decision makers' psychological behavior exhibits risk-averse tendencies for gains and risk-seeking tendencies for losses, that is people are more sensitive to losses than equal gains. For measuring the magnitude of gains and losses, an S-shaped value function is provided in prospect theory (Kahneman and Tversky 1979) (Fig. 2), which shows a prospect value function with a concave and convex S-shape for losses and gains, respectively. The value function is expressed in the form of a power law (Kahneman and Tversky 1979):

$v(x)= \begin{cases}x^{\alpha}, & x \geq 0 \\ -\lambda(-x)^{\beta}, & x<0\end{cases}$

where $x$ denotes the gains with $x \geq 0$ and losses with $x<0$, respectively; $\alpha$ and $\beta$ are power parameters related to gains and losses, respectively; $0 \leq \alpha, \beta \leq 1 . \lambda$ is the risk aversion parameter, which represents a characteristic of being steeper for losses than for gains, $\lambda>1$. The values of $\alpha, \beta$, and $\lambda$ in Eq. 1 are determined through experiments (Abdellaoui et al. 2007; Bleichrodt et al. 2009; Wakker 2010).

To highlight the importance of decision makers' psychological behavior and different emergency situations that need to be dealt with by devising different solutions, several important studies in the literature are notable that are related to our research (Fan et al. 2012; Shu 2012; Liu et al. 2014; Zhou et al. 2014; Qian et al. 2015; Wang et al. 2015; $\mathrm{Yu}$ et al. 2015). These studies have addressed EDM problems from different aspects.

Fan et al. (2012) proposed a risk decision analysis method based on prospect theory for emergency response considering decision makers' psychological behavior. Liu et al. (2014) presented a risk decision analysis method

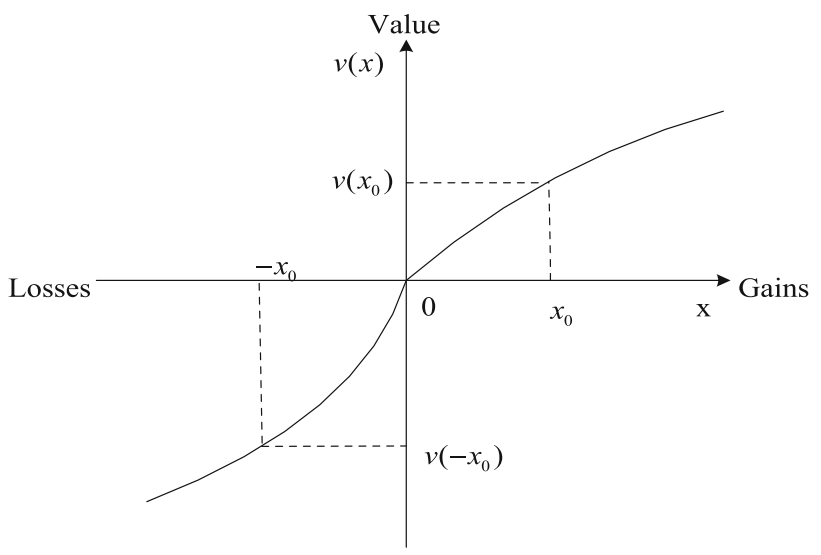

Fig. 2 S-shaped value function of prospect theory

considering decision makers' psychological behavior based on cumulative prospect theory. Wang et al. (2015) proposed a prospect theory-based interval dynamic reference point method for EDM. Qian et al. (2015) proposed a multi-dimensional scenario space method to set up a scenario deduction process with respect to typical oil tank fire cases. Shu (2012) proposed a scenario-response model to deal with the resource allocation and scheduling for unconventional emergencies. Yu et al. (2015) proposed a taxonomy method to design an emergency case pedigree based on scenario-response for emergency events.

Fan et al. (2012), Liu et al. (2014), and Wang et al. (2015) considered decision makers' psychological behavior because of the way they are guided by bounded rationality under risk and uncertainty conditions. However, they dealt with the EDM problems by considering only one situation to select the best alternative. Shu (2012), Zhou et al. (2014), Qian et al. (2015), and Yu et al. (2015) considered the problems regarding different situations in EDM but neglected decision makers' psychological behavior in the decision making process. 


\section{Proposed Method}

This section introduces a novel EDM method based on prospect theory that considers decision makers' psychological behavior and deals with different emergency situations. It consists of six main phases (Fig. 3):

(1) Framework definition: the notations and terminology used in the proposed method are defined.

(2) Information collection: the information related to emergency events (property losses, casualties, environmental effects, and so on) is collected. Based on the collected information, reference points are provided by decision makers regarding different criteria in different possible emergency situations.

(3) Calculation of gains and losses: gains and losses are calculated according to the reference points and predefined amounts of corresponding criteria regarding different alternatives.

(4) Calculation of prospect values: prospect values represent the magnitudes of gains and losses, which reflect the different feelings of decision makers.

(5) Calculation of overall prospect values: the overall prospect value of each alternative is calculated, reflecting the comprehensive performance of each alternative.

(6) Selection of optimal alternatives for different emergency situations: according to the overall prospect value of each alternative, the optimal alternatives for different possible emergency situations are obtained.

These phases are explained in further detail in the following subsections.

\subsection{Framework Definition}

The following notations are used in the proposed method:

- $A=\left\{A_{1}, A_{2}, \ldots, A_{J}\right\}:$ set of alternatives, where $A_{j}$ denotes the $j$-th alternative, $j=1,2, \ldots, J$.

- $S=\left\{S_{1}, S_{2}, \ldots, S_{n}\right\}$ : set of different emergency situations, where $S_{i}$ denotes the $i$-th situation, $i=1,2, \ldots, n$.

- $X=\left\{X_{1}, X_{2}, \ldots, X_{M}\right\}$ : set of criteria/attributes, where $X_{m}$ denotes the $m$-th criterion, $m=1,2, \ldots, M$.

- $C_{j}=\left[C_{j}^{L}, C_{j}^{H}\right], C_{j}^{L} \leq C_{j}^{H}$ : an interval value, where $C_{j}$ denotes the cost of the $j$-th alternative, $j=1,2, \ldots, J$.

- $R_{i m}=\left[R_{i m}^{L}, R_{i m}^{H}\right], R_{i m}^{H}>R_{i m}^{L}$ : an interval value, where $R_{i m}$ denotes the reference point provided by the

Framework definition

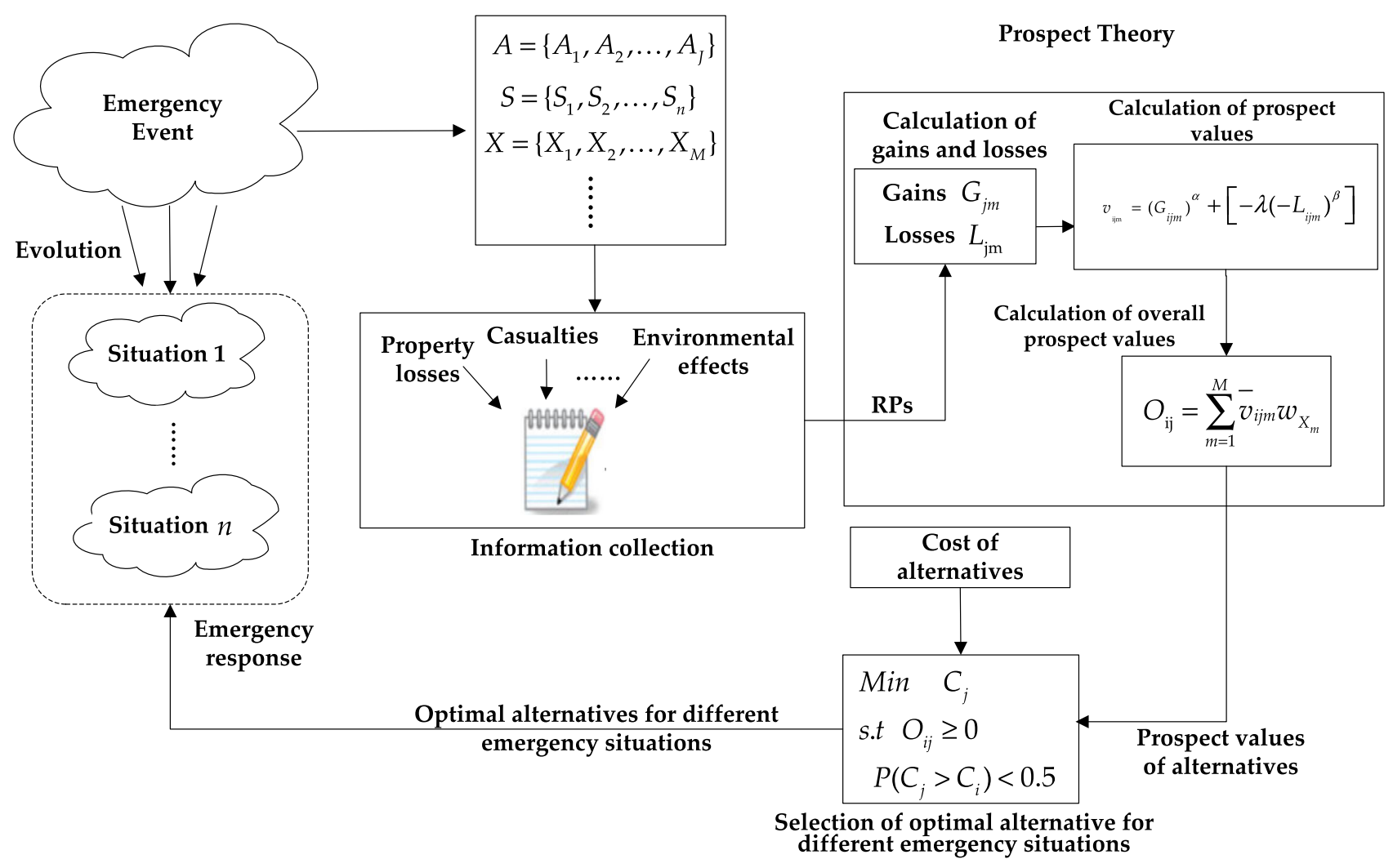

Fig. 3 General framework of the proposed emergency decision making method. ( $R P$ reference point) 
decision maker with respect to $m$-th criterion in the $i$ - $t h$ possible situation, $i=1,2, \ldots, n ; m=1,2, \ldots, M$.

- $E_{j m}=\left[E_{j m}^{L}, E_{j m}^{H}\right], E_{j m}^{H}>E_{j m}^{L}$ : an interval value, where $E_{j m}$ denotes the predefined effective control scope of $j$ th alternative with respect to $m$-th attribute (Wang et al. 2015), which means that the alternative can prevent the losses from the emergency event regarding $X_{m}$, $j=1,2, \ldots, J ; m=1,2, \ldots, M$.

- $W_{X_{m}}=\left(w_{X_{1}}, w_{X_{2}}, \ldots, w_{X_{M}}\right)$ : the weighting vector of criteria, where $w_{X_{m}}$ denotes the criterion weight of $m$ th criterion provided by the decision maker, satisfying $\sum_{m=1}^{M} w_{X_{m}}=1, w_{X_{m}} \in[0,1], m=1,2, \ldots, M$.

\subsection{Information Collection}

When an emergency event occurs, it may evolve into different possible emergency situations because of the dynamic features of emergency events. Before making a decision, it is necessary for the decision maker to collect related information (possible situations, possible losses caused by different possible situations, and so on). According to different possible losses caused by possible emergency situations, the decision maker provides the corresponding reference point, $R_{i m}$, with respect to the $m$-th criterion $X_{m}$ in $i$-th situation $S_{i}$.

In a real world situation, due to inadequate or incomplete information, especially in the early stages of an emergency event, it is difficult for the decision maker to estimate the damages, losses, or costs of emergency alternatives using crisp and precise numbers. Thus, interval values are more suitable for uncertainty modeling (Wang et al. 2015) and were employed in our proposed method.

\subsection{Calculation of Gains and Losses}

Gains and losses reflect decision makers' different psychological behavior, (gains: risk aversion, losses: risk seeking), which are obtained according to the reference point, $R_{i m}$, and the predefined effective control scope, $E_{j m}$, of different emergency alternatives. Because both the reference points and the predefined effective control scope are interval values, the relationship between $R_{i m}$ and $E_{j m}$

Table 1 Possible cases of positional relationship between $R_{i m}$ and $E_{j m}$

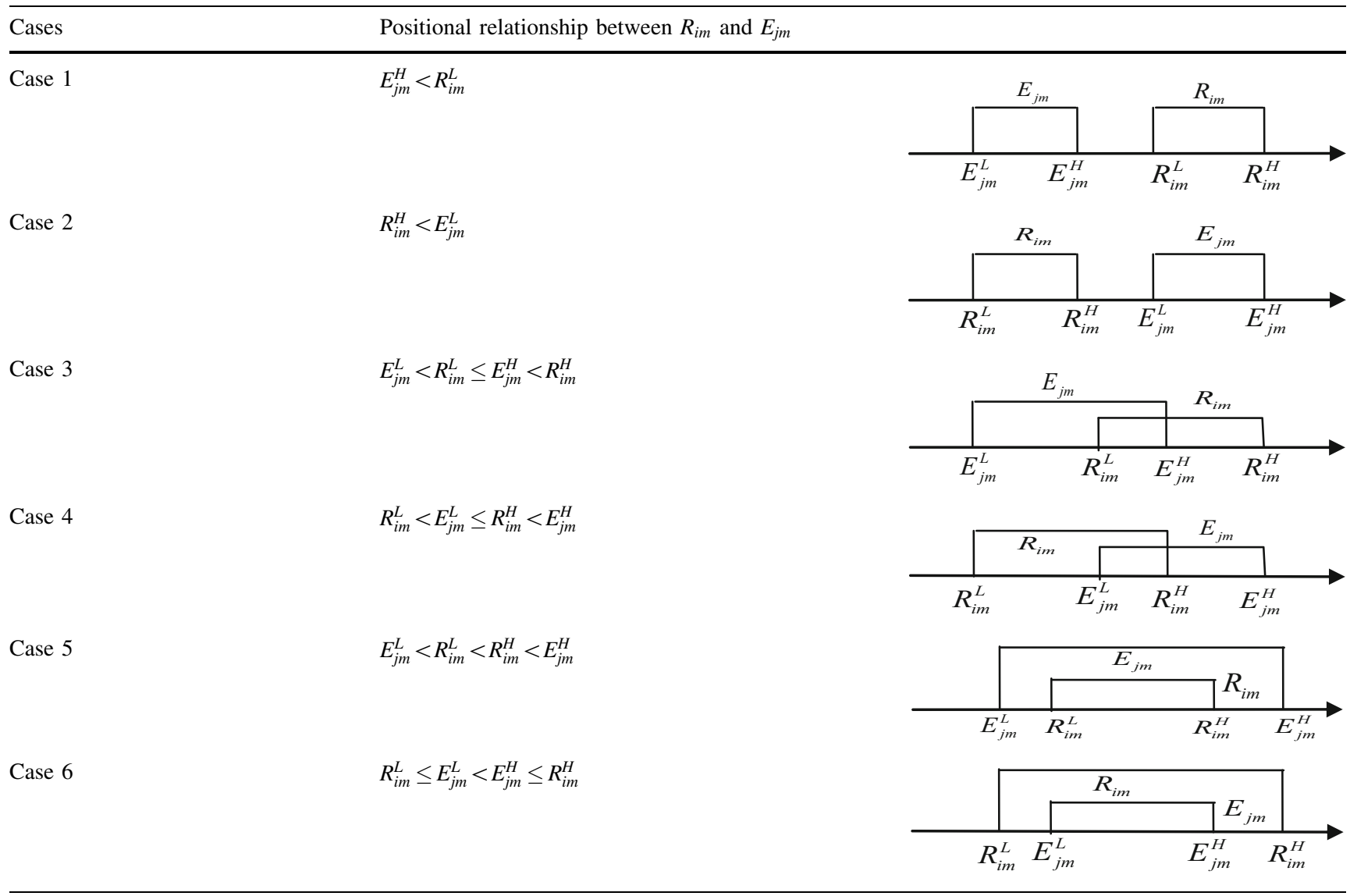


Table 2 Gains and losses for all possible cases (cost criteria)

\begin{tabular}{llll}
\hline Cases & & Gain $G_{j m}$ & \multicolumn{1}{c}{ Loss $L_{j m}$} \\
\hline Case 1 & $E_{j m}^{H}<R_{i m}^{L}$ & $R_{i m}^{L}-0.5\left(E_{j m}^{L}+E_{j m}^{H}\right)$ & 0 \\
Case 2 & $R_{i m}^{H}<E_{j m}^{L}$ & 0 & $R_{i m}^{H}-0.5\left(E_{j m}^{L}+E_{j m}^{H}\right)$ \\
Case 3 & $E_{j m}^{L}<R_{i m}^{L} \leq E_{j m}^{H}<R_{i m}^{H}$ & $0.5\left(R_{i m}^{L}-E_{j m}^{L}\right)$ & 0 \\
Case 4 & $R_{\mathrm{im}}^{L}<E_{j m}^{L} \leq R_{i m}^{H}<E_{j m}^{H}$ & 0 & $0.5\left(R_{i m}^{H}-E_{j m}^{H}\right)$ \\
Case 5 & $E_{j m}^{L}<R_{i m}^{L}<R_{i m}^{H}<E_{j m}^{H}$ & $0.5\left(R_{i m}^{L}-E_{j m}^{L}\right)$ & $0.5\left(R_{i m}^{H}-E_{j m}^{H}\right)$ \\
Case 6 & $R_{i m}^{L} \leq E_{j m}^{L}<E_{j m}^{H} \leq R_{i m}^{H}$ & 0 & 0 \\
\hline
\end{tabular}

Table 3 Gains and losses for all possible cases (benefit criteria)

\begin{tabular}{llll}
\hline Cases & & Gain $G_{j m}$ & \multicolumn{1}{c}{ Loss $L_{j m}$} \\
\hline Case 1 & $E_{j m}^{H}<R_{i m}^{L}$ & 0 & $0.5\left(E_{j m}^{L}+E_{j m}^{H}\right)-R_{i m}^{L}$ \\
Case 2 & $R_{i m}^{H}<E_{j m}^{L}$ & $0.5\left(E_{j m}^{L}+E_{j m}^{H}\right)-R_{i m}^{H}$ & 0 \\
Case 3 & $E_{j m}^{L}<R_{i m}^{L} \leq E_{j m}^{H}<R_{i m}^{H}$ & 0 & $0.5\left(E_{j m}^{L}-R_{i m}^{L}\right)$ \\
Case 4 & $R_{i m}^{L}<E_{j m}^{L} \leq R_{i m}^{H}<E_{j m}^{H}$ & $0.5\left(E_{j m}^{H}-R_{i m}^{H}\right)$ & 0 \\
Case 5 & $E_{j m}^{L}<R_{i m}^{L}<R_{i m}^{H}<E_{j m}^{H}$ & $0.5\left(E_{j m}^{H}-R_{i m}^{H}\right)$ & $0.5\left(E_{j m}^{L}-R_{i m}^{L}\right)$ \\
Case 6 & $R_{i m}^{L} \leq E_{j m}^{L}<E_{j m}^{H} \leq R_{i m}^{H}$ & 0 & 0 \\
\hline
\end{tabular}

should be determined before calculating the gains and losses. The relationship between $R_{i m}$ and $E_{j m}$, and the calculation equations for gains and losses, from Wang et al. (2017) were used in this study.

The positional relationships between $R_{i m}$ and $E_{j m}$ are provided in Table 1 . The calculation equations of gains and losses with our notations for all possible cases are summarized in Tables 2 and 3, with respect to cost and benefit criteria, respectively.

\subsection{Calculation of Prospect Values}

Let $\mathrm{GM}_{i}=\left(G_{i j m}\right)_{J \times M}$ be the gain matrix regarding the $i$-th emergency situation, $\mathrm{LM}_{i}=\left(L_{i j m}\right)_{J \times M}$ be the loss matrix regarding the $i$-th emergency situation. According to prospect theory, the magnitude of gains and losses is measured by value function, let $\mathrm{VM}_{i}=\left(v_{i j m}\right)_{J \times M}$ be the value matrix regarding the $i$-th situation, it can be obtained by using Eq. 2 based on $G M_{i}$ and $L M_{\mathrm{i}}$, that is,

$$
\begin{aligned}
v_{i j m} & =\left(G_{i j m}\right)^{\alpha}+\left[-\lambda\left(L_{i j m}\right)^{\beta}\right], \\
i & =1,2, \ldots, n ; j=1,2, \ldots, J ; m=1,2, \ldots, M
\end{aligned}
$$

where $v_{i j m}$ denotes the value of the $j$-th alternative with respect to the $m$-th criterion in the $i$-th emergency situation. According to Tversky and Kahneman (1992) different values can be used for the parameters of Eq. 2, and we used the following values as the parameters of Eq. 2 in this study, $\alpha=0.89, \beta=0.92, \lambda=2.25$.

Based on Eq. 2, it is easy to obtain the value of $v_{i j m}$. Since the values $v_{i j m}$ are usually incommensurate, they need to be normalized into comparable values. This is achieved by normalizing each element $v_{i j m}$ into a corresponding element in matrix $\overline{V M_{i}}=\left(\bar{v}_{i j m}\right)_{J \times M}$ by using

$\bar{v}_{i j m}=\frac{v_{i j m}}{v_{i j}^{*}}$,

$$
i=1,2, \ldots, n ; j=1,2, \ldots, J ; m=1,2, \ldots, M
$$

where $v_{i j}^{*}=\max _{m \in M}\left\{\left|v_{i j m}\right|\right\}$.

\subsection{Calculation of Overall Prospect Values}

For the sake of simplicity, the attribute weights are provided by the decision maker in this study. By using the simple additive weighting method, the overall prospect value of each alternative can be obtained, that is,

$O_{i j}=\sum_{m=1}^{M} \bar{v}_{i j m} w_{X_{m}}, \quad m=1,2, \ldots, M$

The overall prospect value $O_{i j}$ reflects whether the alternative is adequate for coping with the emergency situation or not. If $O_{i j}>0$, it means that the $j$-th alternative can deal with the $i$-th possible emergency situation. If $O_{i j}<0$, it means that the $j$-th alternative cannot deal with 
the $i$-th possible emergency situation effectively. The bigger $O_{i j}$ is, the better emergency alternative $A_{j}$ will be, and the lower $O_{i j}$ is, the worse emergency alternative $A_{j}$ will be.

\subsection{Selection of Optimal Alternatives for Different Emergency Situations}

According to $O_{i j}$, the ranking of alternatives can be made in a descending order. Based on the existing EDM methods based on prospect theory, the ideal alternative is usually the one with the biggest overall prospect value. However, in the real world, sometimes the ideal alternative is not the optimal one for coping with an emergency event, because there are many other factors that should be taken into account in the alternative selection, such as the cost of an alternative, the quantity of the emergency response resources, and so on. Thus, the overall prospect values of alternatives should not be the only rule to select the ideal alternative for coping with emergency situations. To make the alternative selection close to a real world situation, a linear programing (LP) model is proposed, which considers the cost of alternatives independently to measure the performance of each alternative, that is,

$$
\begin{aligned}
& \text { Min } C_{j} \\
& \text { s.t } \quad O_{i j} \geq 0, \quad i=1,2, \ldots, n ; j=1,2, \ldots, J \\
& \quad P\left(C_{j}>C_{t}\right)<0.5, t, j \in J, j \neq t
\end{aligned}
$$

where $P\left(C_{j}>C_{t}\right)$ denotes the dominance degree that the interval value $C_{j}$ is superior to $C_{t} ; P\left(C_{j}>C_{t}\right)<0.5$ denotes the interval value $C_{j}$ is inferior to $C_{t}$, that is the cost of $j$-th alternative is lower than the cost of $t$-th alternative (for cost of alternatives, the smaller the better). The dominance degree $P\left(C_{j}>C_{t}\right)$ can be calculated by the following equation (Wang et al. 2005):

$$
\begin{aligned}
P\left(C_{j}>C_{t}\right) & =\frac{\max \left(0, \mathrm{C}_{\mathrm{j}}^{\mathrm{H}}-\mathrm{C}_{\mathrm{t}}^{\mathrm{L}}\right)-\max \left(0, \mathrm{C}_{\mathrm{j}}^{\mathrm{L}}-\mathrm{C}_{\mathrm{t}}^{\mathrm{H}}\right)}{\left(C_{j}^{H}-C_{j}^{L}\right)+\left(C_{t}^{H}-C_{t}^{L}\right)}, t, j \\
& \in J, t \neq j
\end{aligned}
$$

According to Eq. 6, $P\left(C_{j}>C_{t}\right)+P\left(C_{t}>C_{j}\right)=1$, and $P\left(C_{j}>C_{t}\right)=P\left(C_{t}>C_{j}\right) \equiv 0.5$ when the interval value $\quad C_{j}=C_{t}$. So, if $P\left(C_{j}>C_{t}\right) \quad$ satisfies $P\left(C_{j}>C_{t}\right)<0.5<P\left(C_{t}>C_{j}\right)$, it is said that $C_{j}$ is inferior to $C_{t}$. It means that the optimal alternative to deal with the $i$-th situation is the alternative $A_{j}$ that satisfies $O_{i j} \geq 0$ with minimum cost.

According to the LP model, the optimal alternative is the one with the minimum cost among the emergency alternatives with $O_{i j} \geq 0$ to deal with the corresponding emergency situation. In summary, the procedures of the proposed method are:
Step 1 Define the framework of the problem

Step 2 The decision maker gathers the related information regarding different possible emergency situations of the emergency event, and provides the corresponding reference points, $R_{i m}$, with respect to each criterion

Step 3 Based on the reference points, $R_{i m}$, and the predefined effective control scope of emergency alternatives, $E_{j m}$, gains and losses are obtained according to Tables 2 and 3. Then, $\mathrm{GM}_{i}$ and $\mathrm{LM}_{i}$ are constructed;

Step 4 Based on $\mathrm{GM}_{i}$ and $\mathrm{LM}_{i}$, the prospect value matrix $\mathrm{VM}_{i}$ can be constructed using Eq. 2, and then normalized $\mathrm{VM}_{i}$ into $\overline{\mathrm{VM}}_{i}$ using Eq. 3

Step 5 The overall prospect value $O_{i j}$ of each alternative is obtained using Eq. 4

Step 6 According to Eqs. 5 and 6, the optimal alternative with respect to each possible emergency situation can be obtained. Then, based on the results, the decision maker can select the optimal alternative for dealing with different emergency situations

\section{Examples of Applying the Proposed Method}

To demonstrate the applicability of the proposed method for dealing with different possible emergency situations, and conduct a fair comparison, two examples of emergency events taken from Wang et al. (2015) - a petrochemical plant fire emergency occurred in a plant of the Sinopec Group of China and a barrier lake emergency caused by the Wenchuan Earthquake that occurred in southwestern China-are presented.

\subsection{Example 1: Petrochemical Plant Fire Emergency}

Petrochemical plant fire is usually characterized by explosibility, diffusivity, and chain reaction. When a petrochemical plant caught fire, it may evolve into different emergency situations and should be dealt with by different solutions. The problem can be solved by the proposed method through the following steps:

Step 1 Framework definition. According to Wang et al. (2015), the following three criteria are concerned in this example

$\mathrm{X}_{1}$ : The number of casualties.

$\mathrm{X}_{2}$ : Property loss (in RMB 10,000 Yuan). 
$\mathrm{X}_{3}$ : Negative effects on the environment on a scale of $0-100$ (0: no negative effect; 100: serious negative effect).

Let $C_{j}$ denotes the cost of the $j$-th alternative (in RMB 10,000 Yuan). From Wang et al. (2015), there are five emergency response alternatives with different effective control scope $E_{j m}$ regarding different criteria and cost $C_{j}$ of each alternative are summarized in Table 4. In addition, the weight of each criterion is provided in parenthesis in Table 4.

Step 2 Information collection. Analyzed by professional experts, there are five possible emergency situations of the petrochemical plant fire:

$S_{1}$ : The local independent production area catches fire;
$S_{2}$ : The storage tanks of different oil products will explode in the local independent production area;

$S_{3}$ : The entire independent production area catches fire;

$S_{4}$ : The nearby production areas catch fire;

$S_{5}$ : The whole petrochemical plant catches fire.

According to the five possible emergency situations, the decision maker provides the reference point, $R_{i m}$, with respect to each emergency situation. All the reference points, $R_{i m}$, with respect to each emergency situation are shown in Table 5.

Step 3 Calculation of gains and losses. According to Tables 4 and 5, the positional relationship between $R_{i m}$ and $E_{j m}$ can be determined based on Table 1, and the $G M_{i}$ and $L M_{i}$ can be constructed based on the equations in Tables 2 and 3, respectively. The $G M_{i}$ and $L M_{i}$ are as follows:

$$
\begin{aligned}
G M_{1}= & {\left[\begin{array}{ccc}
0 & 0 & 10 \\
2.5 & 0 & 20 \\
9 & 50 & 30 \\
17.5 & 450 & 40 \\
32.5 & 1700 & 50
\end{array}\right], G M_{2}=\left[\begin{array}{ccc}
0 & 0 & 0 \\
0 & 0 & 0 \\
2.5 & 0 & 0 \\
10.5 & 350 & 2.5 \\
15.5 & 1600 & 10
\end{array}\right], G M_{3}=\left[\begin{array}{ccc}
0 & 0 & 0 \\
0 & 0 & 0 \\
0 & 0 & 2.5 \\
2.5 & 50 & 10 \\
22.5 & 1100 & 20
\end{array}\right], } \\
G M_{4}= & {\left[\begin{array}{ccc}
0 & 0 & 0 \\
0 & 0 & 5 \\
0 & 0 & 15 \\
0 & 0 & 25 \\
7.5 & 750 & 35
\end{array}\right], G M_{5}=\left[\begin{array}{ccc}
0 & 0 & 0 \\
0 & 0 & 0 \\
0 & 0 & 0 \\
0 & 0 & 15 \\
7.5 & 700 & 25
\end{array}\right], L M_{1}=\left[\begin{array}{ccc}
0 & -25 & 0 \\
0 & 0 & 0 \\
0 & 0 & 0 \\
0 & 0 & 0 \\
0 & 0 & 0
\end{array}\right], L M_{2}=\left[\begin{array}{ccc}
-6 & -225 & -20 \\
-2 & -150 & -10 \\
0 & -50 & -2.5 \\
0 & 0 & 0 \\
-26 & -925 & 0 \\
-14 & -525 & -5 \\
-8.5 & -450 & 0 \\
-2 & -300 & 0 \\
0 & 0 & 0 \\
0 & 0 & 0
\end{array}\right], L M_{4}=\left[\begin{array}{ccc}
-20.5 & -850 & 0 \\
-13 & -700 & 0 \\
-4.5 & -250 & 0 \\
0 & 0 & 0
\end{array}\right], L M_{5}=\left[\begin{array}{ccc}
-20.5 & -1050 \\
-13 & -900 & -2.5 \\
-4.5 & -450 & 0 \\
0 & 0 & 0
\end{array}\right], }
\end{aligned}
$$

Table 4 Predefined effective control scopes, cost of each alternative, and related weights

\begin{tabular}{lllll}
\hline Alternatives & \multicolumn{4}{l}{ Criteria (weights) } \\
\cline { 2 - 5 } & $X_{1}(0.4375)$ & $X_{2}(0.25)$ & $X_{3}(0.3125)$ & $C_{j}$ \\
& $E_{j 1}$ & $E_{j 2}$ & $E_{j 3}$ & $C_{j}$ \\
\hline$A_{1}$ & {$[3,5]$} & {$[50,100]$} & {$[40,50]$} & {$[30,50]$} \\
$A_{2}$ & {$[6,13]$} & {$[100,200]$} & {$[50,60]$} & {$[60,80]$} \\
$A_{3}$ & {$[14,20]$} & {$[200,400]$} & {$[60,70]$} & {$[90,120]$} \\
$A_{4}$ & {$[21,30]$} & {$[500,1000]$} & {$[70,80]$} & {$[130,160]$} \\
$A_{5}$ & {$[31,50]$} & {$[1000,3000]$} & {$[80,90]$} & {$[170,200]$} \\
\hline
\end{tabular}

Step 4 Calculation of prospect values. Based on $G M_{i}$ and $L M_{i}$, the value matrix $V M_{i}$ and its corresponding normalized matrix $\overline{V M_{i}}$ can be constructed by using Eqs. 2 and 3, respectively 
Table 5 Reference points with respect to each emergency situation

\begin{tabular}{llll}
\hline Situations & Criteria & & \\
\cline { 2 - 4 } & $X_{1}$ & $X_{2}$ & $X_{3}$ \\
& $R_{i 1}$ & $R_{i 2}$ & $R_{i 3}$ \\
\hline$S_{1}$ & {$[3,8]$} & {$[100,300]$} & {$[20,35]$} \\
$S_{2}$ & {$[10,15]$} & {$[300,400]$} & {$[65,75]$} \\
$S_{3}$ & {$[18,25]$} & {$[600,900]$} & {$[50,65]$} \\
$S_{4}$ & {$[30,35]$} & {$[1000,1500]$} & {$[40,50]$} \\
$S_{5}$ & {$[30,35]$} & {$[1200,1600]$} & {$[55,60]$} \\
\hline
\end{tabular}

$$
\begin{aligned}
& V M_{1}=\left[\begin{array}{ccc}
0 & -43.48 & 7.76 \\
2.26 & 0 & 14.39 \\
7.07 & 32.51 & 20.64 \\
12.77 & 229.80 & 26.66 \\
22.16 & 750.07 & 32.51
\end{array}\right] \\
& V M_{2}=\left[\begin{array}{ccc}
-11.70 & -328.24 & -35.41 \\
-4.26 & -226.04 & -18.71 \\
2.26 & -82.27 & -5.23 \\
8.11 & 183.75 & 2.26 \\
11.47 & 710.67 & 7.76
\end{array}\right] \text {, } \\
& V M_{3}=\left[\begin{array}{ccc}
-25.50 & -715.70 & -9.89 \\
-16.12 & -621.07 & 0 \\
-4.26 & -427.70 & 2.26 \\
2.26 & 32.51 & 7.76 \\
15.98 & 509.14 & 14.39
\end{array}\right] \text {, } \\
& V M_{4}=\left[\begin{array}{ccc}
-45.08 & -1205.13 & 0 \\
-36.22 & -1114.93 & 4.19 \\
-23.82 & -932.55 & 11.14 \\
-8.98 & -361.65 & 17.55 \\
6.01 & 362.08 & 23.67
\end{array}\right] \text {, } \\
& V M_{5}=\left[\begin{array}{ccc}
-45.08 & -1442.92 & -18.71 \\
-36.22 & -1354.18 & -5.23 \\
-23.82 & -1175.13 & 11.14 \\
-8.98 & -621.07 & 0 \\
6.01 & 340.52 & 17.55
\end{array}\right]
\end{aligned}
$$

and

$$
\begin{aligned}
& \overline{V M}_{1}=\left[\begin{array}{ccc}
0 & -0.0580 & 0.2387 \\
0.1020 & 0 & 0.4424 \\
0.3189 & 0.0433 & 0.6347 \\
0.5764 & 0.3064 & 0.8200 \\
1 & 1 & 1
\end{array}\right], \\
& \overline{V M}_{2}=\left[\begin{array}{ccc}
-1 & -0.4619 & -1 \\
-0.3640 & -0.3181 & -0.5285 \\
0.1932 & -0.1158 & -0.1476 \\
0.6930 & 0.2586 & 0.0638 \\
0.9802 & 1 & 0.2192
\end{array}\right] \text {, } \\
& \overline{V M}_{3}=\left[\begin{array}{ccc}
-1 & -1 & -0.6876 \\
-0.6319 & -0.8678 & 0 \\
-0.1670 & -0.5976 & 0.1571 \\
0.0886 & 0.0454 & 0.5396 \\
0.6263 & 0.7114 & 1
\end{array}\right] \text {, } \\
& \overline{V M}_{4}=\left[\begin{array}{ccc}
-1 & -1 & 0 \\
-0.8036 & -0.9252 & -0.1770 \\
-0.5285 & -0.7738 & 0.4704 \\
-0.1991 & -0.3001 & 0.7412 \\
0.1333 & 0.3004 & 1
\end{array}\right] \text {, } \\
& \overline{V M}_{5}=\left[\begin{array}{ccc}
-1 & -1 & -1 \\
-0.8036 & -0.9385 & -0.2793 \\
-0.5285 & -0.8144 & 0 \\
-0.1991 & -0.4304 & 0.5950 \\
0.1333 & 0.2360 & 0.9375
\end{array}\right]
\end{aligned}
$$

\begin{tabular}{|c|c|c|c|c|c|c|}
\hline \multirow[t]{2}{*}{$O_{i j}$} & & \multicolumn{5}{|l|}{ Situations } \\
\hline & & $S_{1}$ & $S_{2}$ & $S_{3}$ & $S_{4}$ & $S_{5}$ \\
\hline \multirow[t]{5}{*}{ Alternatives (ranking) } & $A_{1}$ & $0.0601(5)$ & $-0.8655(5)$ & $-0.9024(5)$ & $-0.6875(5)$ & $-1.0000(5)$ \\
\hline & $A_{2}$ & $0.1829(4)$ & $-0.4039(4)$ & $-0.4934(4)$ & $-0.5276(4)$ & $-0.6735(4)$ \\
\hline & $A_{3}$ & $0.3487(3)$ & $0.0095(3)$ & $-0.1733(3)$ & $-0.2777(3)$ & $-0.4348(3)$ \\
\hline & $A_{4}$ & $0.5850(2)$ & $0.3878(2)$ & $0.2188(2)$ & $0.0695(2)$ & $-0.0088(2)$ \\
\hline & $A_{5}$ & $1.0000(1)$ & $0.7473(1)$ & $0.7644(1)$ & $0.4459(1)$ & $0.4103(1)$ \\
\hline
\end{tabular}

Step 5 Calculation of overall prospect values. According to Eq. 4 , the overall prospect values, $O_{i j}$, and the corresponding ranking of alternatives with respect to each emergency situation are given in Table 6

From Table 6, the following phenomena can be obtained:

1. For each emergency situation, the alternatives that satisfy $O_{i j} \geq 0$ are more than one, which means that all

Table 6 Overall prospect value of each alternative with respect to each emergency situation 
Table 7 Optimal alternative obtained by our proposed method and by existing emergency decision making (EDM) methods based on prospect theory (PT)

\begin{tabular}{llllll}
\hline & Situations & & & \\
\cline { 2 - 5 } & $S_{1}$ & $S_{2}$ & $S_{3}$ & $S_{4}$ \\
\hline Existing EDM methods based on PT & $A_{5}$ & $A_{5}$ & $A_{5}$ & $A_{5}$ \\
Our proposed method & $A_{1}$ & $A_{3}$ & $A_{4}$ & $A_{4}$ \\
\hline
\end{tabular}

those alternatives satisfying $O_{i j}>0$ can deal with the corresponding emergency situation.

2. From situation $S_{1}$ to $S_{5}$, the numbers of alternatives with $O_{i j} \geq 0$ decrease. This means that different alternatives have different performance regarding different emergency situations.

3. From Table 6, an interesting phenomenon is that according to the selection rule in existing EDM studies based on prospect theory (Fan et al. 2012; Liu et al. 2014; Wang et al. 2015, 2016), the ideal alternative is $A_{5}$ for all emergency situations. This is obviously unreasonable in a real world situation, and $A_{5}$ should not be the only ideal alternative for all emergency situations, because it is easy to result in wasting resources and workforces.

Step 6 Selection of optimal alternatives for different emer gency situations. According to the cost of alternatives shown in Table 4 and the results in Table 6, the optimal alternative can be selected for each possible emergency situation by using Eqs. 5 and 6

According to Eq. 6, it is easy to obtain the following results: $P\left(C_{1}>C_{2}\right)=P\left(C_{2}>C_{3}\right)=P\left(C_{3}>C_{4}\right)=P\left(C_{4}\right.$ $\left.>C_{5}\right)=0<0.5$, that is the ranking of interval values $C_{j}$ is $C_{1} \prec C_{2} \prec C_{3} \prec C_{4} \prec C_{5}$.

Based on Eqs. 5 and 6, the following results are explained.

For situation $S_{1}$, there are five alternatives that satisfy $O_{i j} \geq 0$, that is, $A_{1}, A_{2}, A_{3}, A_{4}$, and $A_{5}$. Among them, the alternative with the minimum cost is $A_{1}$. So, the optimal alternative for dealing with situation $S_{1}$ is $A_{1}$.

For situation $S_{2}$, there are three alternatives that satisfy $O_{i j} \geq 0$, that is, $A_{3}, A_{4}$, and $A_{5}$. Among them, the alternative with the minimum cost is $A_{3}$. So, the optimal alternative for dealing with situation $S_{2}$ is $A_{3}$.

For situation $S_{3}$, there are two alternatives that satisfy $O_{i j} \geq 0$, that is, $A_{4}$ and $A_{5}$. Between $A_{4}$ and $A_{5}$, the alternative with the minimum cost is $A_{4}$. So, the optimal alternative for dealing with situation $S_{3}$ is $A_{4}$.

For situation $S_{4}$, there are two alternatives that satisfy $O_{i j} \geq 0$, that is, $A_{4}$ and $A_{5}$. So like for $S_{3}$, the optimal alternative for dealing with situation $S_{4}$ is $A_{4}$.
For situation $S_{5}$, there is only one alternative that satisfies $O_{i j} \geq 0, A_{5}$. So, the optimal alternative for dealing with situation $S_{5}$ is $A_{5}$.

The results of obtaining optimal alternatives for different possible emergency situations by the proposed method and by existing EDM methods based on prospect theory without considering different emergency situations (Fan et al. 2012; Liu et al. 2014; Wang et al. 2015, 2016) are shown in Table 7.

Table 7 shows that the results obtained by our proposed method are different from the ones obtained by existing EDM methods based on prospect theory because the latter neglect different emergency situations in the EDM process.

\subsection{Example 2: Barrier Lake Emergency}

According to Wang et al. (2015), a barrier lake emergency caused by the Wenchuan Earthquake occurred in southwestern China, which threatened the lives and properties of thousands of people both upstream and downstream. When the barrier lake emergency occurred, the decision maker must take immediate action to avoid people suffering from a disaster. There are usually aftershocks after huge earthquakes and there may be rains, thus the barrier lake emergency might evolve into different situations. Therefore, the barrier lake emergency can be solved by the proposed method through the following steps.

Step 1 Framework definition. The following two criteria cited from Wang et al. (2015) are concerned in this example:

Table 8 Predefined effective control scopes, cost of alternatives, and related weights

\begin{tabular}{llll}
\hline Alternatives & \multicolumn{2}{l}{ Criteria (weights) } \\
\cline { 2 - 4 } & $X_{1}(0.5333)$ & $X_{2}(0.4667)$ & $C_{j}$ \\
& $E_{j 1}$ & $E_{j 2}$ & $C_{j}$ \\
\hline$A_{1}$ & {$[3000,3500]$} & {$[2500,3500]$} & {$[300,350]$} \\
$A_{2}$ & {$[3500,4000]$} & {$[3500,4500]$} & {$[350,450]$} \\
$A_{3}$ & {$[4000,4500]$} & {$[4500,5500]$} & {$[450,550]$} \\
$A_{4}$ & {$[5000,5500]$} & {$[5500,6500]$} & {$[550,650]$} \\
\hline
\end{tabular}


Table 9 Reference points with respect to each emergency situation

\begin{tabular}{llr}
\hline Situations & Criteria & $X_{2}$ \\
\cline { 2 - 3 } & $X_{1}$ & $R_{i 2}$ \\
\hline$S_{1}$ & $R_{i 1}$ & {$[3500,4000]$} \\
$S_{2}$ & {$[2500,3500]$} & {$[4500,5500]$} \\
$S_{3}$ & {$[3000,3500]$} & {$[4000,5500]$} \\
$S_{4}$ & {$[3500,4000]$} & {$[5000,5500]$} \\
\hline
\end{tabular}

$X_{1}$ : The number of people affected.

$X_{2}$ : Property loss (in RMB 10,000 Yuan).

From Wang et al. (2015), there are four emergency response alternatives that can be used to deal with the barrier lake emergency. The effective control scope $E_{j m}$, the cost $C_{j}$ of each alternative are summarized in Table 8 . In addition, the weight of each criterion is provided in parenthesis in Table 8.

Step 2 Information collection. Analyzed by professional experts in hydrological, geological, and meteorological domains, there are four possible emergency situations of the barrier lake in the $72 \mathrm{~h}$ following the emergency

$S_{1}: 1 / 3$ dam body of the barrier lake will break; $S_{2}: 1 / 2$ dam body of the barrier lake will break; $S_{3}: 3 / 4$ dam body of the barrier lake will break; $S_{4}$ : The whole dam body of the barrier lake will break;

According to the four possible emergency situations, the decision maker provides the reference point, $R_{i m}$, with respect to each emergency situation. All the reference points, $R_{i m}$, with respect to each emergency situation are shown in Table 9.

Step 3 Calculation of gains and losses. According to Table 8 and Table 9, the positional relationship between $R_{i m}$ and $E_{j m}$ can be determined based on Table 1, and the $G M_{i}$ and $L M_{i}$ can be constructed based on the equations in Tables 2 and 3, respectively. The $G M_{i}$ and $L M_{i}$ are as follows:

$$
\begin{gathered}
G M_{1}=\left[\begin{array}{cc}
0 & 0 \\
250 & 250 \\
750 & 1000 \\
1750 & 2000
\end{array}\right], G M_{2}=\left[\begin{array}{cc}
0 & 0 \\
250 & 0 \\
750 & 0 \\
1750 & 500
\end{array}\right], \\
G M_{3}=\left[\begin{array}{cc}
0 & 0 \\
0 & 0 \\
250 & 0 \\
1250 & 500
\end{array}\right], G M_{4}=\left[\begin{array}{cc}
0 & 0 \\
0 & 0 \\
0 & 0 \\
250 & 500
\end{array}\right], \\
L M_{1}=\left[\begin{array}{cc}
0 & -500 \\
0 & 0 \\
0 & 0 \\
0 & 0
\end{array}\right], L M_{2}=\left[\begin{array}{cc}
0 & -1500 \\
0 & -1000 \\
0 & -250 \\
0 & 0
\end{array}\right], \\
L M_{3}=\left[\begin{array}{cc}
-250 & -1000 \\
0 & -250 \\
0 & 0 \\
0 & 0
\end{array}\right], L M_{4}=\left[\begin{array}{cc}
-750 & -2000 \\
-250 & -1000 \\
0 & -250 \\
0 & 0
\end{array}\right],
\end{gathered}
$$

Step 4 Calculation of prospect values. Based on $G M_{i}$ and $L M_{i}$, the value matrix $V M_{i}$ and its corresponding normalized matrix $\overline{V M_{i}}$ can be constructed by using Eqs. 2 and 3, respectively 


$$
\begin{aligned}
V M_{1} & =\left[\begin{array}{cc}
0 & -684.28 \\
136.20 & 136.20 \\
362.08 & 467.74 \\
769.67 & 866.80
\end{array}\right], V M_{2}=\left[\begin{array}{cc}
0 & -1880.12 \\
136.20 & -684.28 \\
362.08 & 0 \\
769.67 & 252.40
\end{array}\right], \\
V M_{3}= & {\left[\begin{array}{cc}
-361.65 & -1294.74 \\
0 & -361.65 \\
136.20 & 0 \\
570.49 & 252.40
\end{array}\right], V M_{4}=\left[\begin{array}{cc}
-933.66 & -2449.80 \\
-361.65 & -1294.74 \\
0 & -361.65 \\
136.20 & 252.40
\end{array}\right] }
\end{aligned}
$$

and

$$
\begin{gathered}
\overline{V M}_{1}=\left[\begin{array}{cc}
0 & -0.7894 \\
0.1770 & 0.1571 \\
0.4704 & 0.5396 \\
0 & 0
\end{array}\right], \overline{V M}_{2}=\left[\begin{array}{cc}
0 & -1 \\
0.1770 & -0.3640 \\
0.4704 & 0 \\
1 & 0.1342
\end{array}\right], \\
\overline{V M}_{3}=\left[\begin{array}{cc}
-0.6340 & -1 \\
0 & -0.2793 \\
0.2387 & 0 \\
1 & 0.1949
\end{array}\right], \overline{V M}_{4}=\left[\begin{array}{cc}
-1 & -1 \\
-0.3640 & -0.5285 \\
0 & -0.1476 \\
0.1371 & 0.1030
\end{array}\right]
\end{gathered}
$$

Step 5 Calculation of overall prospect values. According to Eq. 4, the overall prospect values, $O_{i j}$, and the corresponding ranking of alternatives with respect to each emergency situation are given in Table 10

Step 6 Selection of optimal alternatives for different emergency situations. According to the cost of alternatives shown in Table 8 and the results in Table 10, the optimal alternatives for different emergency situations can be determined through Eqs. 5 and 6

According to Eq. 6, it is easy to obtain the following results:

$P\left(C_{1}>C_{2}\right)=P\left(C_{2}>C_{3}\right)=P\left(C_{3}>C_{4}\right)=0<0.5$, that is the ranking of interval values $C_{j}$ is $C_{1} \prec C_{2} \prec C_{3} \prec C_{4}$.

Based on Eqs. 5 and 6, the results are shown in the fourth row of Table 11.

Table 11 shows that the results obtained by our proposed method are different from those obtained by existing EDM methods based on prospect theory.
Table 11 Optimal alternative obtained by our proposed method and by existing emergency decision making (EDM) methods based on prospect theory (PT)

\begin{tabular}{lllll}
\hline Optimal alternative & \multicolumn{4}{l}{ Situations } \\
\cline { 2 - 5 } & $S_{1}$ & $S_{2}$ & $S_{3}$ & $S_{4}$ \\
\hline Existing EDM methods based on PT & $A_{4}$ & $A_{4}$ & $A_{4}$ & $A_{4}$ \\
Our proposed method & $A_{2}$ & $A_{3}$ & $A_{3}$ & $A_{4}$ \\
\hline
\end{tabular}

\section{Conclusion}

This article presents an EDM method based on prospect theory aiming to overcome the limitations of existing approaches. The proposed method considers both decision makers' psychological behavior in the decision making process and different emergency situations, and enriches the existing EDM methods. A linear programing model is applied to obtain more reasonable results than those obtained by existing EDM approaches based on prospect

Table 10 The prospect value of each alternative with respect to each emergency situation

\begin{tabular}{llllll}
\hline$O_{i j}$ & & \multicolumn{2}{l}{ Situations } & \\
\cline { 2 - 5 } & & $S_{1}$ & $S_{2}$ & $S_{3}$ & $S_{4}$ \\
\hline Alternatives (Ranking) & $A_{1}$ & $-0.3684(4)$ & $-0.4667(4)$ & $-0.8048(4)$ & $-1.0000(4)$ \\
& $A_{2}$ & $0.1677(3)$ & $-0.0755(3)$ & $-0.1304(3)$ & $-0.4408(3)$ \\
& $A_{3}$ & $0.5027(2)$ & $0.2509(2)$ & $0.1273(2)$ & $-0.0689(2)$ \\
& $A_{4}$ & $1.0000(1)$ & $0.5960(1)$ & $0.1212(1)$ \\
\hline
\end{tabular}


theory, which takes the overall prospect values as the only alternative selection rule. The proposed method has a simpler and faster computation process than other approaches. It is easy to understand and close to a real world situation. In addition, two examples are provided to illustrate the feasibility and validity of the proposed method. The method developed in this study may have more potential applications in the near future. A promising research direction could be exploring the use of different information types (for example, unknown information, fuzzy linguistic variables and their related types) for EDM under risk and uncertainty conditions.

Acknowledgements This work was partly supported by the Young Doctoral Dissertation Project of the Social Science Planning Project of Fujian Province (Project No. FJ2016C202), and the National Natural Science Foundation of China (Project No. 71371053, 61773123).

Open Access This article is distributed under the terms of the Creative Commons Attribution 4.0 International License (http://crea tivecommons.org/licenses/by/4.0/), which permits unrestricted use, distribution, and reproduction in any medium, provided you give appropriate credit to the original author(s) and the source, provide a link to the Creative Commons license, and indicate if changes were made.

\section{References}

Abdellaoui, M., H. Bleichrodt, and C. Paraschiv. 2007. Loss aversion under prospect theory: A parameter-free measurement. Management Science 53(10): 1659-1674.

Bell, D.E. 1982. Regret in decision making under uncertainty. Operations Research 30(5): 961-981.

Bell, D.E. 1985. Disappointment in decision making under uncertainty. Operations Research 33(1): 1-27.

Bleichrodt, H., U. Schmidt, and H. Zank. 2009. Additive utility in prospect theory. Management Science 55(5): 863-873.

Camerer, C. 1998. Bounded rationality in individual decision making. Experimental Economics 1(2): 163-183.

Cosgrave, J. 1996. Decision making in emergencies. Disaster Prevention and Management: An International Journal 5(4): $28-35$.

Dichtl, H., and W. Drobetz. 2011. Portfolio insurance and prospect theory investors: Popularity and optimal design of capital protected financial products. Journal of Banking \& Finance 35(7): 1683-1697.

Dong, Y., Y. Liu, H. Liang, F. Chiclana, and E. Herrera-Viedma. 2018. Strategic weight manipulation in multiple attribute decision making. Omega 75: 154-164.

Dong, Y., H. Zhang, and E. Herrera-Viedma. 2016. Integrating experts' weights generated dynamically into the consensus reaching process and its applications in managing non-cooperative behaviors. Decision Support Systems 84: 1-15.

Fan, Z.P., Y. Liu, and R.J. Shen. 2012. Risk decision analysis method for emergency response based on prospect theory. Systems Engineering-Theory \& Practice 32(5): 977-984 (in Chinese).

Fan, Z.P., X. Zhang, F.D. Chen, and Y. Liu. 2013. Multiple attribute decision making considering aspiration-levels: A method based on prospect theory. Computers \& Industrial Engineering 65(2): 341-350.
Kahneman, D., and A. Tversky. 1979. Prospect theory: An analysis of decision under risk. Econometrica: Journal of the Econometric Society 47(2): 263-291.

Levy, J.K., and K. Taji. 2007. Group decision support for hazards planning and emergency management: A Group Analytic Network Process (GANP) approach. Mathematical and Computer Modelling 46(7): 906-917.

Li, X.W. 2013. Decision-making method of highway network planning based on prospect theory. Procedia-Social and Behavioral Sciences 96(0): 2042-2050.

Liu, B., X. Zhao, and Y. Li. 2016. Review and prospect of studies on emergency management. Procedia Engineering 145: 1501-1508.

Liu, Y., Z.P. Fan, and Y. Zhang. 2014. Risk decision analysis in emergency response: A method based on cumulative prospect theory. Computers \& Operations Research 42(2): 75-82.

Qian, J., Y. Liu, C. Liu, and Y.Y. Jiao. 2015. Study on case analysis and scenario deduction based on multi-dimensional scenario space method. Systems Engineering-Theory \& Practice 35(10): 2588-2595 (in Chinese).

Schmidt, U., C. Starmer, and R. Sugden. 2008. Third-generation prospect theory. Journal of Risk and Uncertainty 36(3): 203-223.

Schmidt, U., and H. Zank. 2008. Risk aversion in cumulative prospect theory. Management Science 54(1): 208-216.

Schmidt, U., and H. Zank. 2012. A genuine foundation for prospect theory. Journal of Risk and Uncertainty 45(2): 97-113.

Shu, Q. 2012. Resource allocation and scheduling for unconventional emergency based on "scenario-response". Ph.D. dissertation, University of Science and Technology of China, Hefei, China (in Chinese).

Sun, B., W. Ma, B. Li, and X. Li. 2018. Three-way decisions approach to multiple attribute group decision making with linguistic information-based decision-theoretic rough fuzzy set. International Journal of Approximate Reasoning 93: 424-442.

Tversky, A., and D. Kahneman. 1985. The framing of decisions and the psychology of choice. In Environmental impact assessment, technology assessment, and risk analysis, ed. V.T. Covello, J.L. Mumpower, P.J.M. Stallen, and V.R.R. Uppuluri, 107-129. Boston, MA: Springer.

Tversky, A., and D. Kahneman. 1991. Loss aversion in riskless choice: A reference-dependent model. Quarterly Journal of Economics 106(4): 1039-1061.

Tversky, A., and D. Kahneman. 1992. Advances in prospect theory: Cumulative representation of uncertainty. Journal of Risk and Uncertainty 5(4): 297-323.

Wakker, P.P. 2010. Prospect theory: For risk and ambiguity. Cambridge: Cambridge University Press.

Wang, L., Y.M. Wang, and B.X. Hu. 2016. Dynamic adjusting method of emergency alternatives based on prospect theory. Control and Decision 31(1): 99-104 (in Chinese).

Wang, L., Y.M. Wang, and L. Martínez. 2017. A group decision method based on prospect theory for emergency situations. Information Sciences 418: 119-135.

Wang, L., Z.X. Zhang, and Y.M. Wang. 2015. A prospect theorybased interval dynamic reference point method for emergency decision making. Expert Systems with Applications 42(23): 9379-9388.

Wang, Y.M., J.B. Yang, and D.L. Xu. 2005. A preference aggregation method through the estimation of utility intervals. Computers \& Operations Research 32(8): 2027-2049.

Wu, G., and A.B. Markle. 2008. An empirical test of gain-loss separability in prospect theory. Management Science 54(7): $1322-1335$. 
Yu, F., X.Y. Li, and Q.Y. Sun. 2015. Design of the emergency case pedigree for scenario response. Systems Engineering - Theory \& Practice 35(10): 2596-2605 (in Chinese).

Zhang, H., and Y. Liu. 2012. Key problems on fundamental science and technology integration in "scenario-response" based national emergency response platform system. Systems Engineering - Theory \& Practice 32(5): 947-953 (in Chinese).
Zhou, L., X. Wu, Z. Xu, and H. Fujita. 2017. Emergency decision making for natural disasters: An overview. International Journal of Disaster Risk Reduction 27: 567-576.

Zhou, L., S. Zhong, S. Ma, and N. Jia. 2014. Prospect theory based estimation of drivers' risk attitudes in route choice behaviors. Accident Analysis \& Prevention 73: 1-11. 